\title{
Carl Gustav Bjurström traducteur de Claude Simon
}

\section{Carl Gustav Bjurström}

\section{OpenEdition}

Journals

Édition électronique

URL : http://journals.openedition.org/ccs/971

DOI : $10.4000 /$ ccs. 971

ISSN : 2558-782X

\section{Éditeur :}

Presses universitaires de Rennes, Association des lecteurs de Claude Simon

\section{Édition imprimée}

Date de publication : 30 avril 2015

Pagination : 213-219

ISBN : 9782753539990

ISSN : 1774-9425

Référence électronique

Carl Gustav Bjurström, "Carl Gustav Bjurström traducteur de Claude Simon », Cahiers Claude Simon [En ligne], 10 | 2015, mis en ligne le 22 septembre 2017, consulté le 01 mai 2019. URL : http:// journals.openedition.org/ccs/971; DOI : 10.4000/ccs.971 


\section{CARL GUSTAV BJURSTRÖM TRADUCTEUR DE CLAUDE SIMON}

Le 9 novembre 1985, lors des deuxièmes Assises de la traduction littéraire à Arles, une table ronde en présence de Claude Simon et sous la présidence de Lucien Dällenbach a réuni un certain nombre de traducteurs européens de l'ouvre de Simon: John Fletcher, Uffe Harder, Jukka Mannerkorpi, Guido Neri, Helmut Scheffel et Carl Gustav Bjurström. En hommage au grand traducteur suédois et avec l'aimable autorisation de Madame Inger Bjurström et de l'éditeur des actes des Assises, nous reproduisons l'intervention de Carl Gustav Bjurström ${ }^{1}$. On demandait aux traducteurs de commenter deux extraits de l'ouvre: La Bataille de Pharsale, Minuit, p. 176-179 ("bataille quel peintre allemand... Elohim ") et Les Géorgiques, Minuit, p. 118-120 ("Il n'y avait pas de vent non plus... et à la chair des femmes ") (Alastair Duncan).

\section{CARL GUSTAV BJURSTRÖM}

Pour commencer je voudrais dire qu'il y a plusieurs sortes de traductions et plusieurs sortes de traducteurs et qu'un traducteur n'est pas nécessairement apte à traduire tous les textes avec un bonheur égal. Son tempérament le porte plutôt vers tel type de traduction que vers tel autre. Il y a ceux qui aiment à ciseler les aphorismes, ceux qui savent découvrir dans un texte ses origines lointaines, ceux qui s'entourent d'une vaste documentation, vérifiant chaque propos de l'auteur. Il y a ceux qui ont le don de la réplique, il y a les érudits, il y a ceux qui ont l'oreille fine et beaucoup d'autres.

Je ne suis pas un traducteur savant, j'essaie tout bonnement de traduire ce que j'ai sous les yeux. Ce n'est pas par une analyse intellectuelle préliminaire que j'arrive à comprendre ce qu'un texte a dans le ventre, mais en écrivant les mots, en tâchant d'équilibrer les phrases et en m'efforçant de re-produire non pas tant le texte lui-même que le travail de l'auteur, tel qu'il s'est déroulé

1. Actes des deuxièmes Assises de la traduction littéraire, Arles 1985, ATLAS, 1986, p. 95-101. 
dans sa langue. J'aime me sentir le frère d'armes de ceux qui combattent avec leur langue.

Je pense ensuite qu'une traduction est un tout - et que c'est peut-être là sa première difficulté: maintenir un ensemble, ne pas se laisser entraîner ni par les associations souvent divergentes des mots qui, en théorie, correspondent à ceux utilisés par l'auteur, ni par les astuces parfois bien tentantes ou les sonorités trop flatteuses.

Si je suis moins attiré par des œuvres à la respiration courte, élégantes et spirituelles, que par celles qui sont portées par une vaste houle, c'est sans doute aussi parce que les ensembles d'une certaine envergure - le cycle de poèmes plutôt que le poème isolé, le roman plutôt que la nouvelle - obéissent à une économie plus large, plus généreuse, qui autorise des équivalences globales ou décalées. Je veux dire par là qu'un texte peut avoir un certain «ton » qui, dans la langue d'origine, se fait plus particulièrement jour dans certaines tournures, qui n'ont pas d'équivalence directe dans l'autre langue, alors que le "ton" de l'ensemble peut être rendu par d'autres biais. Ou bien que telle astuce de langage, utilisée par l'auteur, peut trouver un écho dans la traduction au détour d'une phrase qui n'est pas nécessairement la même que celle ainsi distinguée dans l'original. Il y a des jeux de mots qui font pour ainsi dire fonction de charnières dans un récit et auquel il faut absolument trouver une équivalence à l'endroit même où ils se trouvent. Il y en a qui dénotent simplement une certaine attitude en face du langage et de ses possibilités et qui ne doivent inciter le traducteur qu'à chercher à prendre lui-même la même attitude, sans essayer de reproduire à chaque instant et au moment même, le jeu de mots de l'original. Quand Queneau parle d'un " chevable vénérable » il est pratiquement impossible de trouver dans chaque langue une traduction exacte, exactement à cet endroit - mais ce n'est pas ce que nous demande le texte de Queneau qui nous incite seulement à faire une pirouette du même genre quand les mots de la langue cible s'y prêtent.

Tout cela pour vous dire que je trouve un peu difficile de parler de la traduction d'extraits, tels qu'on nous en propose. Cela nous invite, j'en ai peur, à une virtuosité plus ou moins gratuite et sans suite véritable, alors que c'est l'ensemble qui compte et les rapports de la partie avec cet ensemble. Des hors-textes en quelque sorte, qui ne permettent pas tout à fait de maintenir la cohérence du texte.

Essayons cependant de commenter ces deux extraits et commençons par Les Géorgiques. C'est probablement le livre le plus difficile que j'aie eu à traduire et cela pour plusieurs raisons. La première est la variation de rythme entre les 
différentes parties, une variation qui n'est pas immédiatement visible, qui n'est pas spectaculaire, mais qui pourtant existe, ce qui fait que pour chaque chapitre il faut commencer de nouveau. Et chacun sait que c'est toujours le début d'une traduction qui est difficile: en fait, on est le plus souvent obligé de le récrire quand on est arrivé à la fin, ce qui entraîne ensuite des rectifications tout au long du texte. Je pense que ce problème se pose également à l'écrivain et lorsque j'ai dit à Claude Simon que ce n'était en réalité qu’après avoir terminé ma traduction que je me sentais enfin capable de la faire, il m’a répondu qu'il en était de même pour lui.

La deuxième est l'insertion de larges extraits des lettres du général, dont le langage doit se distinguer du reste. Ici on se heurte au problème que pose l'évolution d'une langue par rapport à l'autre. Si le français, au XVII ${ }^{\mathrm{e}}$ ou au XVIII ${ }^{\mathrm{e}}$ siècle est beaucoup plus délié que le suédois, moins raide, il est par contre resté plus semblable à lui-même au cours des siècles suivants. Essayer de faire un pastiche du suédois du XVIII ${ }^{e}$ siècle serait donc une erreur et creuserait un abîme trop profond entre les lettres du général et le reste du livre: il ne faut pas que ces lettres deviennent uniquement une curiosité archéologique.

Puis il y a les problèmes inhérents à tous les textes de Claude Simon et qui sont peut-être exacerbés ici.

Le premier paragraphe est un entassement furieux de mots et de phrases, un peu comme lorsque les hommes de corvée jettent des arbres entiers dans le feu. C'est avant tout une question de rythme. Il ne faut pas qu'il soit ralenti, mais il ne faut pas non plus qu'il soit trop saccadé.

Déjà, lorsque l'an dernier nous parlions d'Enfance de Nathalie Sarraute, nous avons rencontré le problème que posent ces séries de mots et de phrases qui se succèdent, sans conjonctions de coordination et uniquement séparés par une virgule - qui ne se prononce pas, mais qui correspond à une respiration. En suédois - et apparemment aussi en anglais - on a tendance à introduire de temps en temps un «et ». Nathalie Sarraute y était opposée, mais s'y est résignée quand ses traducteurs anglais ou suédois lui ont expliqué que c'était nécessaire. Je crois qu'il faut d'une part retenir qu'il y a une différence entre ce qui est énumération et ce qui est précisions successives. Le mot " et ", qui souvent intervient vers la fin d'une énumération pour qu'on retombe sur ses pieds, n'a évidemment pas sa place dans une série de précisions où on reste dans la même catégorie. Je pourrai, si vous le voulez, vous donner des exemples.

D'autre part, si l'on considère le texte que nous avons sous les yeux, il se compose de mots généralement assez longs, mais entre ces mots il y a toute une 
série de mots très courts: le, la, les, de, que, en, etc. Ayant traduit un peu de poésie suédoise en français je sais combien ces mots sont encombrants. Le suédois est une langue agglutinante. Nous ne disons pas "le toit de la maison" mais " hustaket». Lorsque vous essayez de traduire un vers suédois et que vous trouvez en face de cette série de cahots que sont tous ces ne, que, le, en, y, la, c'est à désespérer.

Donc je comprends très bien qu'on essaie dans une phrase française d'éliminer le plus possible de ces mots et entre eux le mot " et ", qui, de plus, est bien plus pointu que le "och" suédois, qui généralement ne se prononce que comme un " $\hat{o}$ ". Je pense donc qu'il faut ici se fier à l'oreille du traducteur pour qu'il produise un rythme correspondant, car seul le rythme permet de comprendre une phrase comme celle-là - et encore est-ce, pour Claude Simon, une phrase d'une brièveté surprenante: même pas une page!

Le second paragraphe casse le rythme, pour repartir de plus belle. Cette rupture ne pose pas de problème. Par contre le participe présent en pose, alternant ici avec le participe passé. Il s'agit ici des deux temps du verbe qui se trouvent ressembler le plus à des adjectifs - en somme l'action réduite à un qualificatif ou le mouvement figé en spectacle. Les hommes n'agissent pas, nous les voyons en train d'agir. Le participe présent - que Claude Simon utilise de façon déjà fort inusitée en France - arrive difficilement à obtenir le même statut en suédois. En français il se termine par une syllabe, "ant ", qui s'évanouit de façon somme toute assez harmonieuse. En suédois il se termine par deux syllabes, "ande ", ce qui fait beaucoup plus monotone, si on l'utilise à longueur de page, et qui en plus me semble légèrement boiteux: "klappande, knakande, tittande », etc. Bien sûr, il y a aussi en suédois des participes présents qui sont devenus des adjectifs ou presque: "blommande, leende, löpande", mais ce ne sont pas des adjectifs actifs, si je peux m'exprimer ainsi. Même le dernier, qui vient du mot " courir ", n'est pas plus actif que lorsque vous parlez en français des "affaires courantes ». Ce qui intéresse dans le participe présent ou passé de Claude Simon, c'est que l'action y est concentrée, gelée, explosive en quelque sorte. Il a donc fallu trouver des tournures qui restituent par d'autres moyens cette expérience de l'action réduite à une qualité. Ces tournures sont multiples et tout dépend du verbe et de sa place dans la phrase, mais je me suis souvent servi de la tournure "Et lui qui faisait ceci ou cela" - qu'on retrouve d'ailleurs souvent dans les romans de Claude Simon ne serait-ce que pour introduire les personnages d'un dialogue: "Et lui » - «Et elle », etc.

Comme pour le mot "et " tout à l'heure, il n'y a pas de solution uniforme, tout dépend du contexte. 
Faut-il s'attarder à quelques détails?

Je remarque, examinant ma traduction avec trois ans de recul, que dans la première phrase, j'ai laissé tomber " il n'y avait ", mais que dans la suivante j'ai introduit un "c'était » avant "comme si » et que j'ai repris la même formule qui sans doute me paraissait donner un élan à la phrase, plus loin: "c'était comme s'ils".

Par contre j'ai laissé tomber "sur place » et surtout "par quelque opération chimique ". Est-ce parce que le mot "particule» me semblait suffisant ou estce parce que je me suis demandé si c'est vraiment par suite d'une opération " chimique " que ces particules s'étaient prises ensemble?

Plus loin j'ai bien répété le mot « démentiel », mais à la fin du paragraphe je n’ai pas répété " gigantesque " qui apparemment m’a paru déranger le rythme en suédois, mais je l'ai remplacé par trois mots, chacun me paraissant s'appliquer plus avantageusement au substantif: bêtes, hommes, forêts: il s'agit sans doute du nombre de syllabes de chacun des adjectifs utilisés.

Notons aussi que le pronom relatif "qui » ou "que " se traduit par le même mot que "comme", ce qui pose parfois quelques problèmes. Je me rappelle m'être un jour plaint à Claude Simon de cette confusion, mais contrairement à mon attente il m'a félicité de pouvoir ainsi écrire en suédois le mot «som » aussi bien pour " comme » que pour " qui ».

Je constate aussi dans le deuxième paragraphe que le suédois m'a offert une très belle image, puisque à la place de "flammes " j'ai pu écrire "eldtungor", c'est-à-dire des langues de feu. Du coup j'ai apparemment préféré les diviser en trois, au lieu de parler de "flammes bifides ", du moment que "partagé en deux " se serait traduit sans indiquer de chiffre par un mot correspondant à " fourchu " ou « fendu ».

Pour finir j’ai préféré écrire " les cimes de l'Olympe " plutôt que « les rochers » - sans doute parce que je m'imaginais le sommet de l'Olympe éclairé par le soleil du matin.

Passons maintenant au deuxième extrait. J'avoue que je m'y sens moins à l'aise, d'une part parce qu'il y a environ quinze ans que j'ai fait cette traduction et que je n'ai pas en mémoire toutes mes motivations, d'autre part parce que j'aime moins l'écriture de ce livre où l'on n'est pas porté par la large houle qu'on trouve dans $L a$ Route des Flandres et Les Géorgiques. Les écritures différentes qui alternent, avec des phrases qui se croisent et se coupent, posent évidemment des problèmes au traducteur qui doit marquer la différence sans faire éclater complètement le texte.

Dans l'ensemble, ces problèmes ne sont pas trop graves. La difficulté la plus importante est bien entendu le passage où Claude Simon commence à jouer avec 
l'orthographe ou à redoubler les syllabes pour aboutir à des mots comme «tragicaca " ou "âmâmour ». On ne peut évidemment pas, dans une autre langue, reproduire une déformation comme "caca de con science ". L'orthographe française, souvent compliquée et ressentie comme un carcan, peut vous inciter à vous livrer comme Queneau, à une écriture entièrement phonétique qui, sur le papier, fait un effet comique. Ainsi par exemple "que faire " écrit K-E-F-E-R. Le suédois, hélas, s'écrit le plus souvent comme il se prononce et il est bien rare qu'on puisse inventer une autre orthographe sans faire disparaittre le mot original. Il est donc difficile de se livrer à ces plaisanteries en suédois et elles apparaissent le plus souvent tirées par les cheveux. Elles ne sont d'ailleurs pas très bonnes dans le texte original, mais c'est évidemment exprès, comme une expression de la rage ou du désir de destruction du personnage. Pour parvenir aux mêmes effets j'ai dû parfois inventer un texte assez différent de celui qui transparaît derrière les déformations de Claude Simon et j'ai dû faire en quelque sorte un travail à rebours, partant du mot grossier ou scatologique, auquel aboutit la déformation de l'orthographe, pour trouver le mot normal qui s'y prêterait et enfin essayer de constituer avec ces mots un texte à peu près cohérent, où l'on retrouverait malgré tout le résumé satirique d'un roman de Graham Greene, La Puissance et la Gloire.

Je vous avoue que le résultat ne me satisfait pas et qu'aucun résultat ici ne pourra sans doute me satisfaire, car en suédois ces déformations prennent un tour irrémédiablement forcé. Dans la mesure où la traduction est réussie, elle devient d'une virtuosité excessive, qui a peu de choses à faire avec le ton de potache rageur de l'original. C'est une des raisons pour lesquelles j'ai tout à l'heure marqué quelque réticence devant l'idée de commenter des extraits et plus particulièrement ce type d'extraits qui présentent des difficultés particulières. La virtuosité ou l'ingéniosité éventuelles avec lesquelles ces difficultés ont été surmontées ne garantissent en rien la qualité de l'ensemble de la traduction et je n'aime pas beaucoup montrer de tels extraits en exemple.

Ces déformations se poursuivent dans le passage suivant, imprimé en italiques, mais à partir de ces italiques, je me suis résolument écarté de l'original, en utilisant une orthographe absolument correcte. En revoyant ce texte, je ne me suis pas tout de suite rendu compte pourquoi j'avais pris cette résolution, puis je me suis aperçu que ce texte en italiques est en fait une citation de Proust, je crois. Et avec ce texte je ne pouvais pas prendre les mêmes libertés qu'avec le texte de Claude Simon qui lui précède - sinon, il perdrait tout à fait sa qualité de citation. Essayer de faire subir au texte de Proust traduit en suédois les mêmes déformations que celles qu'a inventées Claude Simon aurait abouti à un résultat encore plus forcé 
que dans le paragraphe précédent, d'autant que Claude Simon ici est lui-même contraint de se limiter à reproduire le passage de Proust dans une écriture phonétique, qui doit beaucoup à Queneau. Et comme je vous l'ai dit, l'écriture suédoise correcte est déjà en elle-même phonétique.

Je peux cependant vous signaler deux petites choses amusantes, qui se sont passées en dehors de cet extrait et qui me semblent aussi illustrer le travail du traducteur, mais de la même façon ponctuelle. Page 180 on peut lire: " tu as dit Oh bon Dieu Comme tout Bon Dieu ce n'est pas tout de même la première fille que tu Tu parles d'un foin ». Le mot " foin " provient directement du paragraphe précédent où le personnage principal du roman voit par la fenêtre du wagon une charrette de foin. Ici le mot "foin" est bien entendu le mot d'argot pour "bruit ». Il n'est pas possible de trouver en suédois une expression ou un mot qui établisse le même lien avec le paragraphe précédent entre le spectacle vu par la fenêtre et le dialogue des deux hommes. Je me suis permis ici de m'éloigner du texte et de remplacer le mot d'argot par une citation d'Esaïe $(40,6)$ qui prend un certain sens ici, et qui dit "Toute chair est comme l'herbe » avec cette différence que la traduction ancienne - et la plus souvent citée - de la Bible en suédois dit "Allt kött är hö» - "Toute chair est foin ». Il m’a semblé renouer ainsi non seulement avec le paragraphe de la charrette de foin mais aussi avec le livre que lit la voyageuse dans le compartiment et qui est « quelque chose d'anglo-christianosaxon ". C'est là un exemple de ce que j'ai appelé " une équivalence décalée ».

Un peu avant notre extrait, il y a, page 164, un détail qui illustre également le travail du traducteur. Il s'agit d'un avion qui trace sur le ciel le mot ORION et nous y lisons que "l'avion publicitaire entame le dernier jambage de la sixième lettre $\mathrm{N} »$. Or le mot ORION ne comporte que cinq lettres. Avec l'accord de l'auteur - car je crois l'avoir consulté comme je le fais toujours dans des cas pareils - j'ai rectifié dans la traduction suédoise où on lit donc: « le dernier jambage de la cinquième lettre $\mathrm{N} »$. Cela aussi appartient au travail du traducteur:

relever les inconséquences éventuelles du texte et demander à l'auteur si elles sont voulues - auquel cas il faut bien entendu les respecter -, ou si elles sont involontaires et s'il permet alors au traducteur de faire une petite correction. Pour finir, je tiens à dire que tout ce que je viens d'exposer est un raisonnement $a$ posteriori: les choix, les solutions, les observations, les résolutions n'existent pas dans mon esprit avant que je n'entreprenne la traduction. Il n'y a là aucunement une méthode - il n'y a que des commentaires faits après coup. 